\title{
A Method for Symbolic Computation of Abstract Operations ${ }^{\star}$
}

\author{
Aditya Thakur ${ }^{1}$ and Thomas Reps ${ }^{1,2}$ \\ 1 University of Wisconsin, Madison, WI, USA \\ ${ }^{2}$ GrammaTech, Inc.; Ithaca, NY, USA
}

\begin{abstract}
This paper helps to bridge the gap between (i) the use of logic for specifying program semantics and performing program analysis, and (ii) abstract interpretation. Many operations needed by an abstract interpreter can be reduced to the problem of symbolic abstraction: the symbolic abstraction of a formula $\varphi$ in logic $\mathcal{L}$, denoted by $\widehat{\alpha}(\varphi)$, is the most-precise value in abstract domain $\mathcal{A}$ that over-approximates the meaning of $\varphi$. We present a parametric framework that, given $\mathcal{L}$ and $\mathcal{A}$, implements $\widehat{\alpha}$. The algorithm computes successively better overapproximations of $\widehat{\alpha}(\varphi)$. Because it approaches $\widehat{\alpha}(\varphi)$ from "above", if it is taking too much time, a safe answer can be returned at any stage.

Moreover, the framework is "dual-use": in addition to its applications in abstract interpretation, it provides a new way for an SMT (Satisfiability Modulo Theories) solver to perform unsatisfiability checking: given $\varphi \in \mathcal{L}$, the condition $\widehat{\alpha}(\varphi)=\perp$ implies that $\varphi$ is unsatisfiable.
\end{abstract}

\section{Introduction}

This paper concerns the connection between abstract interpretation and logic. Like several previous papers [2937/2112], our work is based on the insight that many of the key operations needed by an abstract interpreter can be reduced to the problem of symbolic abstraction [29].

Suppose that $\mathcal{A}$ is an abstract domain with concretization function $\gamma: \mathcal{A} \rightarrow \mathcal{C}$. Given a formula $\varphi$ in $\operatorname{logic} \mathcal{L}$, let $\llbracket \varphi \rrbracket$ denote the meaning of $\varphi$-i.e., the set of concrete states that satisfy $\varphi$. The symbolic abstraction of $\varphi$, denoted by $\widehat{\alpha}(\varphi)$, is the best $\mathcal{A}$ value that over-approximates $\llbracket \varphi \rrbracket: \widehat{\alpha}(\varphi)$ is the unique value $A \in \mathcal{A}$ such that (i) $\llbracket \varphi \rrbracket \subseteq \gamma(A)$, and (ii) for all $A^{\prime} \in \mathcal{A}$ for which $\llbracket \varphi \rrbracket \gamma\left(A^{\prime}\right), A \sqsubseteq A^{\prime}$.

This paper presents a new framework for performing symbolic abstraction, discusses its properties, and presents several instantiations for various logics and

\footnotetext{
* This research is supported, in part, by NSF under grants CCF- 0810053,0904371$\}$, by ONR under grants N00014-\{09-1-0510, 10-M-0251\}, by ARL under grant W911NF-09-1-0413, by AFRL under grants FA9550-09-1-0279 and FA8650-10-C7088; and by DARPA under cooperative agreement HR0011-12-2-0012. Any opinions, findings, and conclusions or recommendations expressed in this publication are those of the authors, and do not necessarily reflect the views of the sponsoring agencies. T. Reps has an ownership interest in GrammaTech, Inc., which has licensed elements of the technology reported in this publication.
} 
abstract domains. In addition to providing insight on fundamental limits, the new algorithm for $\widehat{\alpha}$ also performs well: our experiments show that it is 11.3 times faster than a competing method [292112, while finding dataflow facts (i.e., invariants) that are equally precise at $76.9 \%$ of a program's basic blocks, better (tighter) at $19.8 \%$ of the blocks, and worse (looser) at only $3.3 \%$ of the blocks.

Most-Precise Abstract Interpretation. Suppose that $\mathcal{G}=\mathcal{C} \stackrel{\gamma}{\leftrightarrows} \mathcal{A}$ is a Galois connection between concrete domain $\mathcal{C}$ and abstract domain $\mathcal{A}$. Then the "best transformer" [7, or best abstract post operator for transition $\tau$, denoted by $\widehat{\operatorname{Post}}[\tau]: \mathcal{A} \rightarrow \mathcal{A}$, is the most-precise abstract operator possible, given $\mathcal{A}$, for the concrete post operator for $\tau, \operatorname{Post}[\tau]: \mathcal{C} \rightarrow \mathcal{C}$. Post $[\tau]$ can be expressed in terms of $\alpha, \gamma$, and $\operatorname{Post}[\tau]$, as follows [7]: $\widehat{\operatorname{Post}}[\tau]=\alpha \circ \operatorname{Post}[\tau] \circ \gamma$. This equation defines the limit of precision obtainable using abstraction $\mathcal{A}$. However, it is nonconstructive; it does not provide an algorithm, either for applying $\widehat{\operatorname{Post}}[\tau]$ or for finding a representation of the function $\widehat{\operatorname{Post}}[\tau]$. In particular, in many cases, the application of $\gamma$ to an abstract value would yield an intermediate result - a set of concrete states - that is either infinite or too large to fit in computer memory.

Symbolic Abstract Operations. The aforementioned problem with applying $\gamma$ can be side-stepped by working with symbolic representations of sets of states (i.e., using formulas in some logic $\mathcal{L}$ ). The use of $\mathcal{L}$ formulas to represent sets of states is convenient because logic can also be used for specifying a language's concrete semantics; i.e., the concrete semantics of a transformer Post $[\tau]$ can be stated as a formula $\varphi_{\tau} \in \mathcal{L}$ that specifies the relation between input states and output states. However, the symbolic approach introduces a new challenge: how to bridge the gap between $\mathcal{L}$ and $\mathcal{A}[29$. In particular, we need to develop (i) algorithms to handle interconversion between formulas of $\mathcal{L}$ and abstract values in $\mathcal{A}$, and (ii) symbolic versions of the operations that form the core repertoire at the heart of an abstract interpreter.

1. $\widehat{\gamma}(A)$ : Given an abstract value $A \in \mathcal{A}$, the symbolic concretization of $A$, denoted by $\widehat{\gamma}(A)$, maps $A$ to a formula $\widehat{\gamma}(A)$ such that $A$ and $\widehat{\gamma}(A)$ represent the same set of concrete states (i.e., $\gamma(A)=\llbracket \widehat{\gamma}(A) \rrbracket)$.

2. $\widehat{\alpha}(\varphi)$ : Given $\varphi \in \mathcal{L}$, the symbolic abstraction of $\varphi$, denoted by $\widehat{\alpha}(\varphi)$, maps $\varphi$ to the best value in $\mathcal{A}$ that over-approximates $\llbracket \varphi \rrbracket$ (i.e., $\widehat{\alpha}(\varphi)=\alpha(\llbracket \varphi \rrbracket)$ ).

3. Assume $[\varphi](A)$ : Given $\varphi \in \mathcal{L}$ and $A \in \mathcal{A}$, Assume $[\varphi](A)$ returns the best value in $\mathcal{A}$ that over-approximates the meaning of $\varphi$ in concrete states described by $A$. That is, Assume $[\varphi](A)$ equals $\alpha(\llbracket \varphi \rrbracket \cap \gamma(A))$.

4. Creation of a representation of $\widehat{\operatorname{Post}}[\tau]$ : Some intraprocedural [15] and many interprocedural 3222 dataflow-analysis algorithms operate on instances of an abstract datatype $\mathcal{T}$ that (i) represents a family of abstract functions (or relations), and (ii) is closed under composition and join. By "creation of a representation of $\widehat{\operatorname{Post}}[\tau]$ ", we mean finding the best instance in $\mathcal{T}$ that over-approximates $\operatorname{Post}[\tau]$.

Several other symbolic abstract operations are discussed in 96 . 
Experience shows that, for most abstract domains, it is easy to write a $\widehat{\gamma}$ function (item 11) 29]. The other three operations are inter-related. $\widehat{\alpha}$ (item [2) can be reduced to Assume (item 3) as follows: $\widehat{\alpha}(\varphi)=\widehat{\operatorname{Assume}}[\varphi](\top)$. Item 4 can be reduced to item 2 as follows: The concrete post operator Post $[\tau]$ corresponds to a formula $\varphi_{\tau} \in \mathcal{L}$ that expresses the transition relation between input states and output states. An instance of abstract datatype $\mathcal{T}$ in item 4 represents an abstract-domain element that denotes an over-approximation of $\llbracket \varphi_{\tau} \rrbracket . \widehat{\alpha}\left(\varphi_{\tau}\right)$ computes the best instance in $\mathcal{T}$ that over-approximates $\llbracket \varphi_{\tau} \rrbracket$.

This paper presents a parametric framework that, for some abstract domains, is capable of performing most-precise abstract operations in the limit. Because the method approaches its result from "above", if the computation takes too much time, it can be stopped to yield a safe result-i.e., an over-approximation to the best abstract operation - at any stage. Thus, the framework provides a tunable algorithm that offers a performance-versus-precision trade-off. We replace "ح" with " " to denote over-approximating operators - e.g., $\widetilde{\alpha}(\varphi)$, $\widetilde{\operatorname{Assume}}[\varphi](A)$, and $\widetilde{\operatorname{Post}}[\tau](A) \widetilde{1}$

Key Insight. In 35, we showed how Stålmarck's method 33, an algorithm for satisfiability checking of propositional formulas, can be explained using abstractinterpretation terminology - in particular, as an instantiation of a more general algorithm, Stålmarck $[\mathcal{A}]$, that is parameterized by a (Boolean) abstract domain $\mathcal{A}$ and operations on $\mathcal{A}$. The algorithm that goes by the name "Stålmarck's method" is one instantiation of Stålmarck $[\mathcal{A}]$ with a certain abstract domain.

Abstract value $A^{\prime}$ is a semantic reduction [7] of $A$ with respect to $\varphi$ if (i) $\gamma\left(A^{\prime}\right) \cap$ $\llbracket \varphi \rrbracket=\gamma(A) \cap \llbracket \varphi \rrbracket$, and (ii) $A^{\prime} \sqsubseteq A$. At each step, Stålmarck $[\mathcal{A}]$ holds some $A \in \mathcal{A}$; each of the so-called "propagation rules" employed in Stålmarck's method improves $A$ by finding a semantic reduction of $A$ with respect to $\varphi$.

The key insight of the present paper is that there is a connection between Stålmarck $[\mathcal{A}]$ and $\widetilde{\alpha}_{\mathcal{A}}$. In essence, to check whether a formula $\varphi$ is unsatisfiable, Stålmarck $[\mathcal{A}]$ computes $\widetilde{\alpha}_{\mathcal{A}}(\varphi)$ and performs the test " $\widetilde{\alpha}_{\mathcal{A}}(\varphi)=\perp_{\mathcal{A}}$ ?" If the test succeeds, it establishes that $\llbracket \varphi \rrbracket \subseteq \gamma\left(\perp_{\mathcal{A}}\right)=\emptyset$, and hence that $\varphi$ is unsatisfiable.

In this paper, we present a generalization of Stålmarck's algorithm to richer logics, such as quantifier-free linear rational arithmetic (QF_LRA) and quantifierfree bit-vector arithmetic (QF_BV). Instead of only using a Boolean abstract domain, the generalized method of this paper also uses richer abstract domains, such as the polyhedral domain 8 and the bit-vector affine-relations domain [12]. By this means, we obtain algorithms for computing $\widetilde{\alpha}$ for these richer abstract domains. The bottom line is that our algorithm is "dual-use": (i) it can be used by an abstract interpreter to compute $\widetilde{\alpha}$ (and perform other symbolic abstract operations), and (ii) it can be used in an SMT (Satisfiability Modulo Theories) solver to determine whether a formula is satisfiable.

$1 \widetilde{\operatorname{Post}}[\tau]$ is used by Graf and Saïdi $[14$ to mean a different state transformer from the one that $\widetilde{\text { Post }}[\tau]$ denotes in this paper. Throughout the paper, we use $\widetilde{\text { Post }}[\tau]$ solely to mean an over-approximation of $\widehat{\operatorname{Post}}[\tau]$; thus, our notation is not ambiguous. 
Because we are working with more expressive logics, our algorithm uses several ideas that go beyond what is used in either Stålmarck's method [33] or in Stålmarck $[\mathcal{A}]$ [35. The methods described in this paper are also quite different from the huge amount of recent work that uses decision procedures in program analysis. It has become standard to reduce program paths to formulas by encoding a program's actions in logic (e.g., by symbolic execution) and calling a decision procedure to determine whether a given path through the program is feasible. In contrast, the techniques described in this paper adopt-and adapt - the key ideas from Stålmarck's method to create new algorithms for key program-analysis operations. Finally, the methods described in this paper are quite different from previous methods for symbolic abstraction [29 37/21/12], which all make repeated calls to an SMT solver.

Contributions. The contributions of the paper can be summarized as follows:

- We present a connection between symbolic abstraction and Stålmarck's method for checking satisfiability (\$2).

- We present a generalization of Stålmarck's method that lifts the algorithm from propositional logic to richer logics (\$3).

- We present a new parametric framework that, for some abstract domains, is capable of performing most-precise abstract operations in the limit, including $\widehat{\alpha}(\varphi)$ and $\widehat{A s s u m e}[\varphi](A)$, as well as creating a representation of $\widehat{\operatorname{Post}}[\tau]$. Because the method approaches most-precise values from "above", if the computation takes too much time it can be stopped to yield a sound result.

- We present instantiations of our framework for two logic/abstract-domain pairs: QF_BV/KS and QF_LRA/Polyhedra, and discuss completeness (\$4).

- We present experimental results that illustrate the dual-use nature of our framework. One experiment uses it to compute abstract transformers, which are then used to generate invariants; another experiment uses it for checking satisfiability (\$5).

$\$ 6$ discusses other symbolic operations. 97 discusses related work. Proofs can be found in 36.

\section{Overview}

We now illustrate the key points of our Stålmarck-inspired technique using two examples. The first shows how our technique applies to computing abstract transformers; the second describes its application to checking unsatisfiability.

The top-level, overall goal of Stålmarck's method can be understood in terms of the operation $\widetilde{\alpha}(\psi)$. However, Stålmarck's method is recursive (counting down on a parameter $k$ ), and the operation performed at each recursive level is the slightly more general operation Assume $[\psi](A)$. Thus, we will discuss Assume.

Example 1. Consider the following x86 assembly code

$$
\text { L1: cmp eax, } 2 \text { L2: jz L4 L3: ... }
$$

The instruction at L1 sets the zero flag (zf) to true if the value of register eax equals 2. At instruction L2, if $\mathrm{zf}$ is true the program jumps to location L4 (not 
seen in the code snippet) by updating the value of the program counter (pc) to L4; otherwise, control falls through to program location L3. The transition formula that expresses the state transformation from the beginning of L1 to the beginning of L4 is thus $\varphi=(\mathrm{zf} \Leftrightarrow(\mathrm{eax}=2)) \wedge\left(\mathrm{pc}^{\prime}=\operatorname{ITE}(\mathrm{zf}, \mathrm{L} 4, \mathrm{~L} 3)\right) \wedge\left(\mathrm{pc}^{\prime}=\right.$ L4) $\wedge\left(\operatorname{eax}^{\prime}=\operatorname{eax}\right)$. ( $\varphi$ is a QF_BV formula.)

Let $\mathcal{A}$ be the abstract domain of affine relations over the x86 registers. Let $A_{0}=\top_{\mathcal{A}}$, the empty set of affine constraints over input-state and output-state variables. We now describe how our algorithm creates a representation of the $\mathcal{A}$ transformer for $\varphi$ by computing Assume $[\varphi]\left(A_{0}\right)$. The result represents a sound abstract transformer for use in affine-relation analysis (ARA) [27/21/2]. First, the ITE term in $\varphi$ is rewritten as $\left(z f \Rightarrow\left(p c^{\prime}=\mathrm{L} 4\right)\right) \wedge\left(\neg \mathrm{zf} \Rightarrow\left(p c^{\prime}=\mathrm{L} 3\right)\right)$. Thus, the transition formula becomes $\varphi=(\mathrm{zf} \Leftrightarrow(\operatorname{eax}=2)) \wedge\left(\mathrm{zf} \Rightarrow\left(\mathrm{pc}^{\prime}=\mathrm{L} 4\right)\right) \wedge$ $\left(\neg \mathrm{zf} \Rightarrow\left(\mathrm{pc} c^{\prime}=\mathrm{L} 3\right)\right) \wedge\left(\mathrm{pc}^{\prime}=\mathrm{L} 4\right) \wedge\left(\mathrm{eax}^{\prime}=\mathrm{eax}\right)$

Next, propagation rules are used to compute a semantic reduction with respect to $\varphi$, starting from $A_{0}$. The main feature of the propagation rules is that they are "local"; that is, they make use of only a small part of formula $\varphi$ to compute the semantic reduction.

1. Because $\varphi$ has to be true, we can conclude that each of the conjuncts of $\varphi$ are also true; that is, $\mathrm{zf} \Leftrightarrow(\mathrm{eax}=2), \mathrm{zf} \Rightarrow\left(\mathrm{pc}^{\prime}=\mathrm{L} 4\right), \neg \mathrm{zf} \Rightarrow\left(\mathrm{pc}^{\prime}=\mathrm{L3}\right)$, $\mathrm{pc}^{\prime}=\mathrm{L} 4$, and $\mathrm{eax}^{\prime}=$ eax are all true.

2. Suppose that we have a function $\mu \widetilde{\alpha}_{\mathcal{A}}$ such that for a literal $l \in \mathcal{L}, A^{\prime}=$ $\mu \widetilde{\alpha}_{\mathcal{A}}(l)$ is a sound overapproximation of $\widehat{\alpha}(l)$. Because the literal $\mathrm{pc}^{\prime}=\mathrm{L} 4$ is true, we conclude that $A^{\prime}=\mu \widetilde{\alpha}_{\mathcal{A}}\left(\mathrm{pc}^{\prime}=\mathrm{L} 4\right)=\left\{\mathrm{pc}^{\prime}-\mathrm{L} 4=0\right\}$ holds, and thus $A_{1}=A_{0} \sqcap A^{\prime}=\left\{\mathrm{pc}^{\prime}-\mathrm{L} 4=0\right\}$, which is a semantic reduction of $A_{0}$.

3. Similarly, because the literal eax' ${ }^{\prime}=$ eax is true, we obtain $A_{2}=A_{1} \sqcap$ $\mu \widetilde{\alpha}_{\mathcal{A}}\left(\operatorname{eax}^{\prime}=\operatorname{eax}\right)=\left\{\mathrm{pc}^{\prime}-\mathrm{L} 4=0, \operatorname{eax}^{\prime}-\operatorname{eax}=0\right\}$.

4. We know that $\neg \mathbf{z f} \Rightarrow\left(\mathrm{pc}^{\prime}=\mathrm{L} 3\right)$. Furthermore, $\mu \widetilde{\alpha}_{\mathcal{A}}\left(\mathrm{pc} c^{\prime}=\mathrm{L} 3\right)=\left\{\mathrm{pc} c^{\prime}-\mathrm{L} 3=\right.$ $0\}$. Now $\left\{\mathrm{pc}^{\prime}-\mathrm{L} 3=0\right\} \sqcap A_{2}$ is $\perp$, which implies that $\llbracket \mathrm{pc}^{\prime}=\mathrm{L} 3 \rrbracket \cap \gamma\left(\left\{\mathrm{pc} \mathrm{c}^{\prime}-\mathrm{L} 4=\right.\right.$ 0 , eax $\left.\left.{ }^{\prime}-\operatorname{eax}=0\right\}\right)=\emptyset$. Thus, we can conclude that $\neg \mathbf{z f}$ is false, and hence that $\mathbf{z f}$ is true. This value of $\mathbf{z f}$, along with the fact that $\mathbf{z f} \Leftrightarrow(\operatorname{eax}=2)$ is true, enables us to determine that $A^{\prime \prime}=\mu \widetilde{\alpha}_{\mathcal{A}}($ eax $=2)=\{$ eax $-2=0\}$ must hold. Thus, our final semantic-reduction step produces $A_{3}=A_{2} \sqcap A^{\prime \prime}=$ $\left\{\mathrm{pc}^{\prime}-\mathrm{L} 4=0, \mathrm{eax}^{\prime}-\mathrm{eax}=0, \mathrm{eax}-2=0\right\}$.

Abstract value $A_{3}$ is a set of affine constraints over the registers at L1 (inputstate variables) and those at L4 (output-state variables), and can be used for affine-relation analysis using standard techniques (e.g., see [19] or [12, §5]).

The above example illustrates how our technique propagates truth values to various subformulas of $\varphi$. The process of repeatedly applying propagation rules to compute Assume is called 0-assume. The next example illustrates the Dilemma Rule, a more powerful rule for computing semantic reductions.

Example 2. Let $\mathcal{L}$ be QF_LRA, and let $\mathcal{A}$ be the polyhedral abstract domain [8]. Consider the formula $\psi=\left(a_{0}<b_{0}\right) \wedge\left(a_{0}<c_{0}\right) \wedge\left(b_{0}<a_{1} \vee c_{0}<a_{1}\right) \wedge\left(a_{1}<\right.$ $\left.b_{1}\right) \wedge\left(a_{1}<c_{1}\right) \wedge\left(b_{1}<a_{2} \vee c_{2}<a_{2}\right) \wedge\left(a_{2}<a_{0}\right) \in \mathcal{L}$ (see Fig. 1(a)). Suppose that we want to compute Assume $[\psi]\left(\top_{\mathcal{A}}\right)$. 


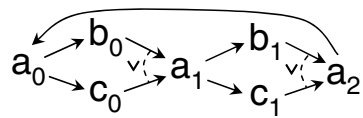

(a)

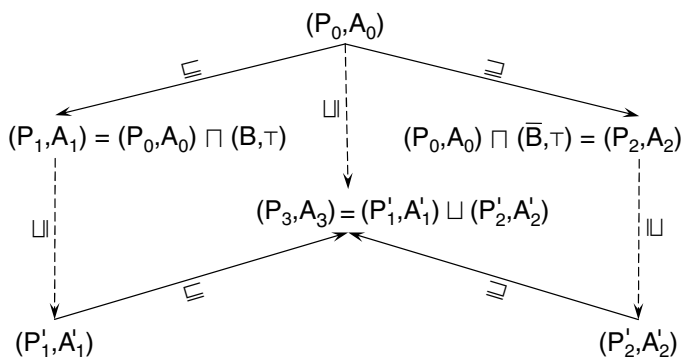

(b)

Fig. 1. (a) Inconsistent inequalities in the (unsatisfiable) formula used in Ex. 2. (b) Application of the Dilemma Rule to abstract value $\left(P_{0}, A_{0}\right)$. The dashed arrows from $\left(P_{i}, A_{i}\right)$ to $\left(P_{i}^{\prime}, A_{i}^{\prime}\right)$ indicate that $\left(P_{i}^{\prime}, A_{i}^{\prime}\right)$ is a semantic reduction of $\left(P_{i}, A_{i}\right)$.

To make the communication between the truth values of subformulas and the abstract value explicit, we associate a fresh Boolean variable with each subformula of $\psi$ to give a set of integrity constraints $\mathcal{I}$. In this case, $\mathcal{I}_{\psi}=\left\{u_{1} \Leftrightarrow \bigwedge_{i=2}^{8} u_{i}, u_{2} \Leftrightarrow\left(a_{0}<b_{0}\right), u_{3} \Leftrightarrow\left(a_{0}<c_{0}\right), u_{4} \Leftrightarrow\left(u_{9} \vee u_{10}\right), u_{5} \Leftrightarrow\left(a_{1}<\right.\right.$ $\left.b_{1}\right), u_{6} \Leftrightarrow\left(a_{1}<c_{1}\right), u_{7} \Leftrightarrow\left(u_{11} \vee u_{12}\right), u_{8} \Leftrightarrow\left(a_{2}<a_{0}\right), u_{9} \Leftrightarrow\left(b_{0}<a_{1}\right), u_{10} \Leftrightarrow\left(c_{0}<\right.$ $\left.\left.a_{1}\right), u_{11} \Leftrightarrow\left(b_{1}<a_{2}\right), u_{12} \Leftrightarrow\left(c_{1}<a_{2}\right)\right\}$. The integrity constraints encode the structure of $\psi$ via the set of Boolean variables $\mathcal{U}=\left\{u_{1}, u_{2}, \ldots, u_{12}\right\}$. When $\mathcal{I}$ is used as a formula, it denotes the conjunction of the individual integrity constraints.

We now introduce an abstraction over $\mathcal{U}$; in particular, we use the Cartesian domain $\mathcal{P}=(\mathcal{U} \rightarrow\{0,1, *\})_{\perp}$ in which $*$ denotes "unknown", and each element in $\mathcal{P}$ represents a set of assignments in $\mathbb{P}(\mathcal{U} \rightarrow\{0,1\})$. We denote an element of the Cartesian domain as a mapping, e.g., $\left[u_{1} \mapsto 0, u_{2} \mapsto 1, u_{3} \mapsto *\right]$, or $[0,1, *]$ if $u_{1}, u_{2}$, and $u_{3}$ are understood. $\top_{\mathcal{P}}$ is the element $\lambda u . *$. The "single-point" partial assignment in which variable $v$ is set to $b$ is denoted by $\top_{\mathcal{P}}[v \mapsto b]$.

The variable $u_{1} \in \mathcal{U}$ represents the root of $\psi$; consequently, the single-point partial assignment $\top_{\mathcal{P}}\left[u_{1} \mapsto 1\right]$ corresponds to the assertion that $\psi$ is satisfiable. In fact, the models of $\psi$ are closely related to the concrete values in $\llbracket \mathcal{I} \rrbracket \cap$ $\gamma\left(\top_{\mathcal{P}}\left[u_{1} \mapsto 1\right]\right)$. For every concrete value in $\llbracket \mathcal{I} \rrbracket \cap \gamma\left(\top_{\mathcal{P}}\left[u_{1} \mapsto 1\right]\right)$, its projection onto $\left\{a_{i}, b_{i}, c_{i} \mid 0 \leq i \leq 1\right\} \cup\left\{a_{2}\right\}$ gives us a model of $\psi$; that is, $\llbracket \psi \rrbracket=(\llbracket \mathcal{I} \rrbracket \cap$ $\left.\gamma\left(\top_{\mathcal{P}}\left[u_{1} \mapsto 1\right]\right)\right)\left.\right|_{\left(\left\{a_{i}, b_{i}, c_{i} \mid 0 \leq i \leq 1\right\} \cup\left\{a_{2}\right\}\right)}$. By this means, the problem of computing Assume $[\psi]\left(\top_{\mathcal{A}}\right)$ is reduced to that of computing Assume $[\mathcal{I}]\left(\left(\top_{\mathcal{P}}\left[u_{1} \mapsto 1\right], \top_{\mathcal{A}}\right)\right)$, where $\left(\top_{\mathcal{P}}\left[u_{1} \mapsto 1\right], \top_{\mathcal{A}}\right)$ is an element of the reduced product of $\mathcal{P}$ and $\mathcal{A}$.

Because $u_{1}$ is true in $\top_{\mathcal{P}}\left[u_{1} \mapsto 1\right]$, the integrity constraint $u_{1} \Leftrightarrow \bigwedge_{i=2}^{8} u_{i}$ implies that $u_{2} \ldots u_{8}$ are also true, which refines $\top_{\mathcal{P}}\left[u_{1} \mapsto 1\right]$ to $P_{0}=$ $[1,1,1,1,1,1,1,1, *, *, *, *]$. Because $u_{2}$ is true and $u_{2} \Leftrightarrow\left(a_{0}<b_{0}\right) \in \mathcal{I}, \top_{\mathcal{A}}$ can be refined using $\mu \widetilde{\alpha}_{\mathcal{A}}\left(a_{0}<b_{0}\right)=\left\{a_{0}-b_{0}<0\right\}$. Doing the same for $u_{3}, u_{5}, u_{6}$, and $u_{8}$, refines $\top_{\mathcal{A}}$ to $A_{0}=\left\{a_{0}-b_{0}<0, a_{0}-c_{0}<0, a_{1}-b_{1}<0, a_{1}-c_{1}<0, a_{2}-a_{0}<0\right\}$. These steps refine $\left(\top_{\mathcal{P}}\left[u_{1} \mapsto 1\right], \top_{\mathcal{A}}\right)$ to $\left(P_{0}, A_{0}\right)$ via 0 -assume.

To increase precision, we need to use the Dilemma Rule, a branch-and-merge rule, in which the current abstract state is split into two (disjoint) abstract states, 0 -assume is applied to both abstract values, and the resulting abstract 
values are merged by performing a join. The steps of the Dilemma Rule are shown schematically in Fig. 1(b) and described below.

In our example, the value of $u_{9}$ is unknown in $P_{0}$. Let $B \in \mathcal{P}$ be $\top_{\mathcal{P}}\left[u_{9} \mapsto 0\right]$; then $\bar{B}$, the abstract complement of $B$, is $\top_{\mathcal{P}}\left[u_{9} \mapsto 1\right]$. Note that $\gamma(B) \cap \gamma(\bar{B})=\emptyset$, and $\gamma(B) \cup \gamma(\bar{B})=\gamma(\top)$. The current abstract value $\left(P_{0}, A_{0}\right)$ is split into

$$
\left(P_{1}, A_{1}\right)=\left(P_{0}, A_{0}\right) \sqcap(B, \top) \quad \text { and } \quad\left(P_{2}, A_{2}\right)=\left(P_{0}, A_{0}\right) \sqcap(\bar{B}, \top) .
$$

Now consider 0 -assume on $\left(P_{1}, A_{1}\right)$. Because $u_{9}$ is false, and $u_{4}$ is true, we can conclude that $u_{10}$ has to be true, using the integrity constraint $u_{4} \Leftrightarrow\left(u_{9} \vee u_{10}\right)$. Because $u_{10}$ holds and $u_{10} \Leftrightarrow\left(c_{0}<a_{1}\right) \in \mathcal{I}, A_{1}$ can be refined with the constraint $c_{0}-a_{1}<0$. Because $a_{0}-c_{0}<0 \in A_{1}, a_{0}-a_{1}<0$ can be inferred. Similarly, when performing 0 -assume on $\left(P_{2}, A_{2}\right), a_{0}-a_{1}<0$ is inferred. Call the abstract values computed by 0 -assume $\left(P_{1}^{\prime}, A_{1}^{\prime}\right)$ and $\left(P_{2}^{\prime}, A_{2}^{\prime}\right)$, respectively. At this point, the join of $\left(P_{1}^{\prime}, A_{1}^{\prime}\right)$ and $\left(P_{2}^{\prime}, A_{2}^{\prime}\right)$ is taken. Because $a_{0}-a_{1}<0$ is present in both branches, it is retained in the join. The resulting abstract value is $\left(P_{3}, A_{3}\right)=$ $\left([1,1,1,1,1,1,1,1, *, *, *, *],\left\{a_{0}-b_{0}<0, a_{0}-c_{0}<0, a_{1}-b_{1}<0, a_{1}-c_{1}<\right.\right.$ $\left.0, a_{2}-a_{0}<0, a_{0}-a_{1}<0\right\}$. Note that although $P_{3}$ equals $P_{0}, A_{3}$ is strictly more precise than $A_{0}$ (i.e., $\left.A_{3} \sqsubset A_{0}\right)$, and hence $\left(P_{3}, A_{3}\right)$ is a semantic reduction of $\left(P_{0}, A_{0}\right)$ with respect to $\psi$.

Now suppose $\left(P_{3}, A_{3}\right)$ is split using $u_{11}$. Using reasoning similar to that performed above, $a_{1}-a_{2}<0$ is inferred on both branches, and hence so is $a_{0}-a_{2}<0$. However, $a_{0}-a_{2}<0$ contradicts $a_{2}-a_{0}<0$; consequently, the abstract value reduces to $\left(\perp_{\mathcal{P}}, \perp_{\mathcal{A}}\right)$ on both branches. Thus, Assume $[\psi]\left(\top_{\mathcal{A}}\right)=\perp_{\mathcal{A}}$, and hence $\psi$ is unsatisfiable. In this way, Assume instantiated with the polyhedral domain can be used to decide the satisfiability of a QF_LRA formula.

The process of repeatedly applying the Dilemma Rule is called 1-assume. That is, repeatedly some variable $u \in \mathcal{U}$ is selected whose truth value is unknown, the current abstract value is split using $B=\top_{\mathcal{P}}[u \mapsto 0]$ and $\bar{B}=\top_{\mathcal{P}}[u \mapsto 1]$, 0 -assume is applied to each of these values, and the resulting abstract values are merged via join (Fig. 1(b)). Different policies for selecting the next variable on which to split can affect how quickly an answer is found; however, any fair selection policy will return the same answer. The efficacy of the Dilemma Rule is partially due to case-splitting; however, the real power of the Dilemma Rule is due to the fact that it preserves information learned in both branches when a case-split is "abandoned" at a join point.

The generalization of the 1-assume algorithm is called k-assume: repeatedly some variable $u \in \mathcal{U}$ is selected whose truth value is unknown, the current abstract value is split using $B=\top_{\mathcal{P}}[u \mapsto 0]$ and $\bar{B}=\top_{\mathcal{P}}[u \mapsto 1] ;(\mathrm{k}-1)$-assume is applied to each of these values; and the resulting values are merged via join. However, there is a trade-off: higher values of $k$ give greater precision, but are also computationally more expensive.

For certain abstract domains and logics, Assume $[\psi]\left(\top_{\mathcal{A}}\right)$ is complete -i.e., with a high-enough value of $k$ for k-assume, Assume $[\psi]\left(\top_{\mathcal{A}}\right)$ always computes the most-precise $\mathcal{A}$ value possible for $\psi$. However, our experiments show that Assume $[\psi]\left(\top_{\mathcal{A}}\right)$ has very good precision with $k=1$ (see $\$ 5$ ) - which jibes with the observation that, in practice, with Stålmarck's method for propositional 


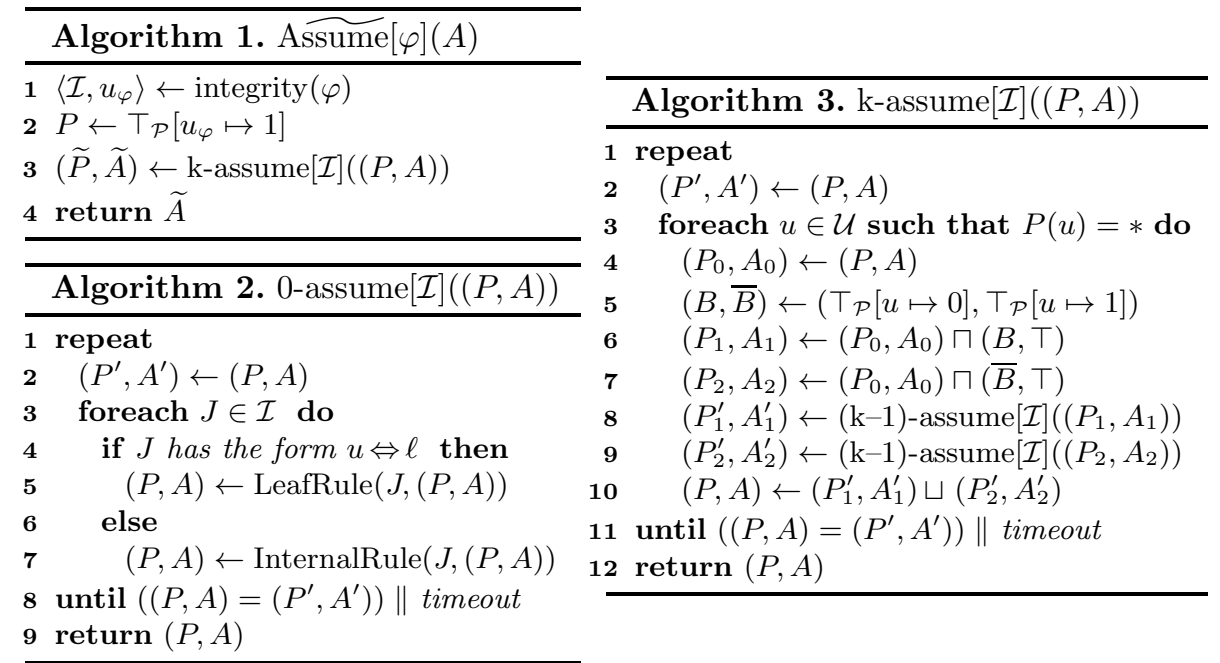

validity (tautology) checking "a formula is either [provable with $k=1$ ] or not a tautology at all!" [18, p. 227].

\section{Algorithm for Assume $[\varphi](A)$}

This section presents our algorithm for computing $\widetilde{\operatorname{ssume}}[\varphi](A) \in \mathcal{A}$, for $\varphi \in \mathcal{L}$. The assumptions of our framework are as follows:

1. There is a Galois connection $\mathcal{C} \underset{\alpha}{\stackrel{\gamma}{\leftrightarrows}} \mathcal{A}$ between $\mathcal{A}$ and concrete domain $\mathcal{C}$.

2. There is an algorithm to perform the join of arbitrary elements of $\mathcal{A}$.

3. Given a literal $l \in \mathcal{L}$, there is an algorithm $\mu \widetilde{\alpha}$ to compute a safe (overapproximating) "micro- $\widetilde{\alpha}$ "-i.e., $A^{\prime}=\mu \widetilde{\alpha}(l)$ such that $\gamma\left(A^{\prime}\right) \supseteq \llbracket l \rrbracket$.

4. There is an algorithm to perform the meet of an arbitrary element of $\mathcal{A}$ with an arbitrary element of $\{\mu \widetilde{\alpha}(l) \mid \ell \in \operatorname{literal}(\mathcal{L})\}$.

Note that $\mathcal{A}$ is allowed to have infinite descending chains; because Assume works from above, it is allowed to stop at any time, and the value in hand is an overapproximation of the most precise answer.

Alg. 1 presents the algorithm that computes Assume $[\varphi](A)$ for $\varphi \in \mathcal{L}$ and $A \in \mathcal{A}$. Line (10) calls the function integrity, which converts $\varphi$ into integrity constraints $\mathcal{I}$ by assigning a fresh Boolean variable to each subformula of $\varphi$, using the rules described in Fig. 2. The variable $u_{\varphi}$ corresponds to formula $\varphi$. We use $\mathcal{U}$ to denote the set of Boolean variables created when converting $\varphi$ to $\mathcal{I}$. Alg. 1 also uses a second abstract domain $\mathcal{P}$, each of whose elements represents a set of Boolean assignments in $\mathbb{P}(\mathcal{U} \rightarrow\{0,1\})$. For simplicity, in this paper $\mathcal{P}$ is the Cartesian domain $(\mathcal{U} \rightarrow\{0,1, *\})_{\perp}$, but other more-expressive Boolean domains could be used [35].

On line (2) of Alg. 11 an element of $\mathcal{P}$ is created in which $u_{\varphi}$ is assigned the value 1 , which asserts that $\varphi$ is true. Alg. 1 is parameterized by the value of $k$ (where $k \geq 0)$. Let $\gamma_{\mathcal{I}}((P, A))$ denote $\gamma((P, A)) \cap \llbracket \mathcal{I} \rrbracket$. The call to k-assume on 


$$
\frac{\varphi:=\ell \quad \ell \in \operatorname{literal}(\mathcal{L})}{u_{\varphi} \Leftrightarrow \ell \in \mathcal{I}} \text { LEAF }
$$

$$
\frac{\varphi:=\varphi_{1} \text { op } \varphi_{2}}{u_{\varphi} \Leftrightarrow\left(u_{\varphi_{1}} \text { op } u_{\varphi_{2}}\right) \in \mathcal{I}} \text { INTERNAL }
$$

Fig. 2. Rules used to convert a formula $\varphi \in \mathcal{L}$ into a set of integrity constraints $\mathcal{I}$. op represents any binary connective in $\mathcal{L}$, and $\operatorname{literal}(\mathcal{L})$ is the set of atomic formulas and their negations.

line (3) returns $(\widetilde{P}, \widetilde{A})$, which is a semantic reduction of $(P, A)$ with respect to $\mathcal{I}$; that is, $\gamma_{\mathcal{I}}((\widetilde{P}, \widetilde{A}))=\gamma_{\mathcal{I}}((P, A))$ and $(\widetilde{P}, \widetilde{A}) \sqsubseteq(P, A)$. In general, the greater the value of $k$, the more precise is the result computed by Alg. 11. The next theorem states that Alg. 1 computes an over-approximation of Assume $[\varphi](A)$.

Theorem 1 ([36]). For all $\varphi \in \mathcal{L}, A \in \mathcal{A}$, if $\widetilde{A}=\widehat{A s s u m e}[\varphi](A)$, then $\gamma(\widetilde{A}) \supseteq$ $\llbracket \varphi \rrbracket \cap \gamma(A)$, and $\widetilde{A} \sqsubseteq A$.

Alg. 3 presents the algorithm to compute k-assume, for $k \geq 1$. Given the integrity constraints $\mathcal{I}$, and the current abstract value $(P, A)$, k-assume $[\mathcal{I}]((P, A))$ returns an abstract value that is a semantic reduction of $(P, A)$ with respect to $\mathcal{I}$. The crux of the computation is the inner loop body, lines (41)-(10), which implements an analog of the Dilemma Rule from Stålmarck's method 33.

The steps of the Dilemma Rule are shown schematically in Fig. 1(b). At line (3) of Alg. 3, a Boolean variable $u$ whose value is unknown is chosen. $B=$ $\top_{\mathcal{P}}[u \mapsto 0]$ and its complement $\bar{B}=\top_{\mathcal{P}}[u \mapsto 1]$ are used to split the current abstract value $\left(P_{0}, A_{0}\right)$ into two abstract values $\left(P_{1}, A_{1}\right)=(P, A) \sqcap(B, \top)$ and $\left(P_{2}, A_{2}\right)=(P, A) \sqcap(\bar{B}, \top)$, as shown in lines (6) and (7).

The calls to $(\mathrm{k}-1)$-assume at lines (8) and (9) compute semantic reductions of $\left(P_{1}, A_{1}\right)$ and $\left(P_{2}, A_{2}\right)$ with respect to $\mathcal{I}$, which creates $\left(P_{1}^{\prime}, A_{1}^{\prime}\right)$ and $\left(P_{2}^{\prime}, A_{2}^{\prime}\right)$, respectively. Finally, at line (10) $\left(P_{1}^{\prime}, A_{1}^{\prime}\right)$ and $\left(P_{2}^{\prime}, A_{2}^{\prime}\right)$ are merged by performing a join. (The result is labeled $\left(P_{3}, A_{3}\right)$ in Fig. 1 (b).)

The steps of the Dilemma Rule (Fig. 1(b)) are repeated until a fixpoint is reached, or some resource bound is exceeded. The next theorem states that k-assume $[\mathcal{I}]((P, A))$ computes a semantic reduction of $(P, A)$ with respect to $\mathcal{I}$.

Theorem 2 ([36]). For all $P \in \mathcal{P}$ and $A \in \mathcal{A}$, if $\left(P^{\prime}, A^{\prime}\right)=$ k-assume $[\mathcal{I}]((P, A))$, then $\gamma_{\mathcal{I}}\left(\left(P^{\prime}, A^{\prime}\right)\right)=\gamma_{\mathcal{I}}((P, A))$ and $\left(P^{\prime}, A^{\prime}\right) \sqsubseteq(P, A)$.

Alg. 2 describes the algorithm to compute 0-assume: given the integrity constraints $\mathcal{I}$, and an abstract value $(P, A), 0$-assume $[\mathcal{I}]((P, A))$ returns an abstract value $\left(P^{\prime}, A^{\prime}\right)$ that is a semantic reduction of $(P, A)$ with respect to $\mathcal{I}$. It is in this algorithm that information is passed between the component abstract values $P \in \mathcal{P}$ and $A \in \mathcal{A}$ via propagation rules, like the ones shown in Figs. 3 and 4. In lines (4)-(7) of Alg. 2, these rules are applied by using a single integrity constraint in $\mathcal{I}$ and the current abstract value $(P, A)$.

Given $J \in \mathcal{I}$ and $(P, A)$, the net effect of applying any of the propagation rules is to compute a semantic reduction of $(P, A)$ with respect to $J \in \mathcal{I}$. The propagation rules used in Alg. 2 can be classified into two categories: 


$$
\begin{aligned}
& \frac{J=\left(u_{1} \Leftrightarrow\left(u_{2} \vee u_{3}\right)\right) \in \mathcal{I} \quad P\left(u_{1}\right)=0}{\left(P \sqcap \top\left[u_{2} \mapsto 0, u_{3} \mapsto 0\right], A\right)} \text { OR1 } \\
& \frac{J=\left(u_{1} \Leftrightarrow\left(u_{2} \wedge u_{3}\right)\right) \in \mathcal{I} \quad P\left(u_{1}\right)=1}{\left(P \sqcap \top\left[u_{2} \mapsto 1, u_{3} \mapsto 1\right], A\right)} \text { AND1 }
\end{aligned}
$$

Fig. 3. Boolean rules used by Alg. 2 in the call InternalRule $(J,(P, A))$

$$
\begin{gathered}
\frac{J=(u \Leftrightarrow l) \in \mathcal{I} \quad P(u)=1}{\left(P, A \sqcap \mu \widetilde{\alpha}_{\mathcal{A}}(l)\right)} \text { PтоA-1 } \quad \frac{J=(u \Leftrightarrow l) \in \mathcal{I} \quad P(u)=0}{\left(P, A \sqcap \mu \widetilde{\alpha}_{\mathcal{A}}(\neg l)\right)} \text { PтоA-0 } \\
\frac{J=(u \Leftrightarrow \ell) \in \mathcal{I} \quad A \sqcap \mu \widetilde{\alpha}_{\mathcal{A}}(l)=\perp_{\mathcal{A}}}{(P \sqcap \top[u \mapsto 0], A)} \text { ATOP-0 }
\end{gathered}
$$

Fig. 4. Rules used by Alg. 2 in the call LeafRule $(J,(P, A))$

1. Rules that apply on line (7) when $J$ is of the form $p \Leftrightarrow(q$ op $r)$, shown in Fig. 3. Such an integrity constraint is generated from each internal subformula of formula $\varphi$. These rules compute a non-trivial semantic reduction of $P$ with respect to $J$ by only using information from $P$. For instance, rule And1 says that if $J$ is of the form $p \Leftrightarrow(q \wedge r)$, and $p$ is 1 in $P$, then we can infer that both $q$ and $r$ must be 1 . Thus, $P \sqcap \top[q \mapsto 1, r \mapsto 1]$ is a semantic reduction of $P$ with respect to $J$. (See Ex. 1, step 1)

2. Rules that apply on line (5) when $J$ is of the form $u \Leftrightarrow \ell$, shown in Fig. 4 . Such an integrity constraint is generated from each leaf of the original formula $\varphi$. This category of rules can be further subdivided into

(a) Rules that propagate information from abstract value $P$ to abstract value $A$; viz., rules PтоA-0 and PтоA-1. For instance, rule $\mathrm{P}$ тоA-1 states that given $J=u \Leftrightarrow l$, and $P(u)=1$, then $A \sqcap \mu \widetilde{\alpha}(l)$ is a semantic reduction of $A$ with respect to $J$. (See Ex. 1, steps 2 and 3.)

(b) Rule AтоP-0, which propagates information from abstract value $A$ to abstract value $P$. Rule AтOP-0 states that if $J=(u \Leftrightarrow \ell)$ and $A \sqcap \mu \widetilde{\alpha}(l)=$ $\perp_{\mathcal{A}}$, then we can infer that $u$ is false. Thus, the value of $P \sqcap \top[u \mapsto 0]$ is a semantic reduction of $P$ with respect to $J$. (See Ex. 1] step 4.)

Alg. 2 repeatedly applies the propagation rules until a fixpoint is reached, or some resource bound is reached. The next theorem states that 0 -assume computes a semantic reduction of $(P, A)$ with respect to $\mathcal{I}$.

Theorem 3 ([36]). For all $P \in \mathcal{P}, A \in \mathcal{A}$, if $\left(P^{\prime}, A^{\prime}\right)=0$-assume $[\mathcal{I}]((P, A))$, then $\gamma_{\mathcal{I}}\left(\left(P^{\prime}, A^{\prime}\right)\right)=\gamma_{\mathcal{I}}((P, A))$ and $\left(P^{\prime}, A^{\prime}\right) \sqsubseteq(P, A)$.

\section{Instantiations}

In this section, we describe instantiations of our framework for two logicallanguage/abstract-domain pairs: QF_BV/KS and QF_LRA/Polyhedra. We say 
that an Assume algorithm is complete for a logic $\mathcal{L}$ and abstract domain $\mathcal{A}$ if it is guaranteed to compute the best value $\widehat{\operatorname{Assume}}[\varphi](A)$ for $\varphi \in \mathcal{L}$ and $A \in \mathcal{A}$. We give conditions under which the two instantiations are complete.

Bitvector Affine-Relation Domain (QF_BV/KS). King and Søndergaard 21] gave an algorithm for $\widehat{\alpha}$ for an abstract domain of Boolean affine relations. Elder et al. 12 extended the algorithm to arithmetic modulo $2^{w}$ (i.e., bitvectors of width $w$ ). Both algorithms work from below, making repeated calls on a SAT solver (King and Søndergaard) or an SMT solver (Elder et al.), performing joins to create increasingly better approximations of the desired answer. We call this family of domains KS, and call the (generalized) algorithm $\widehat{\alpha}_{\mathrm{KS}}^{\uparrow}$.

Given a literal $l \in$ QF_BV, we compute $\mu \widetilde{\alpha}_{\mathrm{KS}}(l)$ by invoking $\widehat{\alpha}_{\mathrm{KS}}^{\uparrow}(l)$. That is, we harness $\widehat{\alpha}_{\mathrm{KS}}^{\uparrow}$ in service of $\widetilde{\mathrm{Assume}}_{\mathrm{KS}}$, but only for $\mu \widetilde{\alpha}_{\mathrm{KS}}$, which means that $\widehat{\alpha}_{\mathrm{KS}}^{\uparrow}$ is only applied to literals. If an invocation of $\widehat{\alpha}_{\mathrm{KS}}^{\uparrow}$ does not return an answer within a specified time limit, we use $T_{K S}$.

Alg. 1 is not complete for QF_BV/KS. Let $x$ be a bitvector of width 2 , and let $\varphi=(x \neq 0 \wedge x \neq 1 \wedge x \neq 2)$. Thus, $\widehat{A s s u m e}[\varphi]\left(\top_{\mathrm{KS}}\right)=\{x-3=0\}$. The KS domain is not expressive enough to represent disequalities. For instance, $\mu \widetilde{\alpha}(x \neq 0)$ equals $\top_{\text {KS }}$. Because Alg. 1 considers only a single integrity constraint at a time, we get Assume $[\varphi]\left(\top_{\mathrm{KS}}\right)=\mu \widetilde{\alpha}(x \neq 0) \sqcap \mu \widetilde{\alpha}(x \neq 1) \sqcap \mu \widetilde{\alpha}(x \neq 2)=\top_{K S}$.

The current approach can be made complete for QF_BV/KS by making 0 -assume consider multiple integrity constraints during propagation (in the limit, having to call $\mu \widetilde{\alpha}(\varphi))$. For the affine subset of QF_BV, an alternative approach would be to perform a $2^{w}$-way split on the KS value each time a disequality is encountered, where $w$ is the bit-width-in effect, rewriting $x \neq 0$ to $(x=1 \vee x=2 \vee x=3)$. Furthermore, if there is a $\mu$ Assume operation, then the second approach can be extended to handle all of QF_BV: $\mu$ Assume $[\ell](A)$ would be used to take the current KS abstract value $A$ and a literal $\ell$, and return an over-approximation of Assume $[\ell](A)$. All these approaches would be prohibitively expensive. Our current approach, though theoretically not complete, works very well in practice (see \$5).

Polyhedral Domain (QF_LRA/Polyhedra). The second instantiation that we implemented is for the logic QF_LRA and the polyhedral domain [8]. Because a QF_LRA disequality $t \neq 0$ can be normalized to $(t<0 \vee t>0)$, every literal $l$ in a normalized QF_LRA formula is merely a half-space in the polyhedral domain. Consequently, $\mu \widetilde{\alpha}_{\text {Polyhedra }}(l)$ is exact, and easy to compute. Furthermore, because of this precision, the Assume algorithm is complete for QF_LRA/Polyhedra. In particular, if $k=|\varphi|$, then k-assume is sufficient to guarantee that $\widehat{\operatorname{Assume}}[\varphi](A)$ returns Assume $[\varphi](A)$. For polyhedra, our implementation uses PPL [28].

The observation in the last paragraph applies in general: if $\mu \widetilde{\alpha}_{\mathcal{A}}(l)$ is exact for all literals $l \in \mathcal{L}$, then Alg. 11 is complete for logic $\mathcal{L}$ and abstract domain $\mathcal{A}$.

\section{Experiments}

Bitvector Affine-Relation Analysis (ARA). We compare two methods for computing the abstract transformers for the KS domain for ARA [21]: 


\begin{tabular}{|c|c|c|c|c|c|c|c|c|}
\hline \multirow{2}{*}{$\begin{array}{l}\text { Prog. } \\
\text { name }\end{array}$} & \multicolumn{4}{|c|}{ Measures of size } & \multicolumn{4}{|c|}{$\widehat{\alpha}^{\uparrow}$ Performance } \\
\hline & instrs & CFGs & BBs & brs & WPDS & $\mathrm{t} / \mathrm{o}$ & post $^{*}$ & query \\
\hline finger & 532 & 18 & 298 & 48 & 110.9 & & 0.266 & 0.0 \\
\hline & 1093 & 16 & 609 & 74 & 204.4 & & 0.344 & 0.016 \\
\hline & 1167 & 16 & 573 & 103 & 148.9 & 2 & 0.344 & 0.032 \\
\hline dsk & 1468 & 18 & 787 & 119 & 384.4 & 16 & 0.219 & 0.031 \\
\hline vert & 1927 & 38 & 1013 & 161 & 289.9 & 9 & 1.047 & 0.062 \\
\hline & 1982 & 40 & 931 & 243 & 562.9 & 14 & 1.281 & 0.046 \\
\hline & 2470 & 46 & 1145 & 306 & 621.1 & 16 & 1.938 & 0.063 \\
\hline setup & 4751 & 67 & 1862 & 589 & 1524.7 & 64 & 0.968 & 0.047 \\
\hline
\end{tabular}

Fig. 5. WPDS experiments $\left(\widehat{\alpha}^{\uparrow}\right)$. The columns show the number of instructions (instrs); the number of procedures (CFGs); the number of basic blocks (BBs); the number of branch instructions (brs); the times, in seconds, for WPDS construction with $\widehat{\alpha}_{\mathrm{KS}}^{\uparrow}$ weights, running post ${ }^{*}$, and finding one-vocabulary affine relations at blocks that end with branch instructions (query). The number of basic blocks for which $\widehat{\alpha}_{\mathrm{KS}}^{\uparrow}$-weight generation timed out is listed under "t/o".

- the $\widehat{\alpha}^{\uparrow}$-based procedure described in Elder et al. [12.

- the $\widetilde{\alpha}$-based procedure described in this paper ( $\left.\widetilde{\alpha}{ }^{\downarrow} "\right)$, instantiated for KS.

Our experiments were designed to answer the following questions:

1. How does the speed of $\widetilde{\alpha}^{\downarrow}$ compare with that of $\widehat{\alpha}^{\uparrow}$ ?

2. How does the precision of $\widetilde{\alpha}^{\downarrow}$ compare with that of $\widehat{\alpha}^{\uparrow}$ ?

To address these questions, we performed ARA on x86 machine code, computing affine relations over the $\mathrm{x} 86$ registers. Our experiments were run on a single core of a quad-core $3.0 \mathrm{GHz}$ Xeon computer running 64-bit Windows XP (SP2), configured so that a user process has $4 \mathrm{~GB}$ of memory. We analyzed a corpus of Windows utilities using the WALi 20 system for weighted pushdown systems (WPDSs). For the baseline $\widehat{\alpha}^{\uparrow}$-based analysis we used a weight domain of $\widehat{\alpha}^{\uparrow}$ generated KS transformers. The weight on each WPDS rule encodes the KS transformer for a basic block $B$ of the program, including a jump or branch to a successor block. A formula $\varphi_{B}$ is created that captures the concrete semantics of $B$, and then the KS weight for $B$ is obtained by performing $\widehat{\alpha}^{\uparrow}\left(\varphi_{B}\right)$ (cf. Ex. 1). We used EWPDS merge functions [24 to preserve caller-save and callee-save registers across call sites. The post* query used the FWPDS algorithm 23.

Fig. 5 lists several size parameters of the examples (number of instructions, procedures, basic blocks, and branches) along with the times for constructing abstract transformers and running post ${ }^{*}$ Col. 6 of Fig. 5 shows that the calls to $\widehat{\alpha}^{\uparrow}$ during WPDS construction dominate the total time for ARA.

Each call to $\widehat{\alpha}^{\uparrow}$ involves repeated invocations of an SMT solver. Although the overall time taken by $\widehat{\alpha}^{\uparrow}$ is not limited by a timeout, we use a 3 -second timeout for each invocation of the SMT solver (as in Elder et al. [12]). Fig. 5 lists the number of such SMT solver timeouts for each benchmark. In case the invocation

${ }^{2}$ Due to the high cost of the $\widehat{\alpha}^{\uparrow}$-based WPDS construction, all analyses excluded the code for libraries. Because register eax holds the return value from a call, library functions were modeled approximately (albeit unsoundly, in general) by "havoc(eax)". 


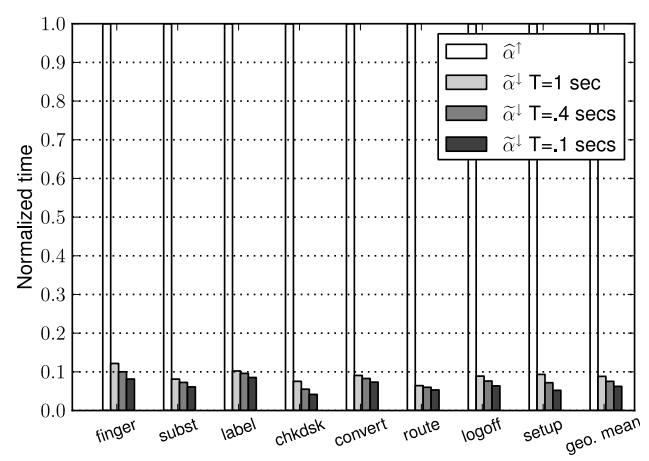

(a)

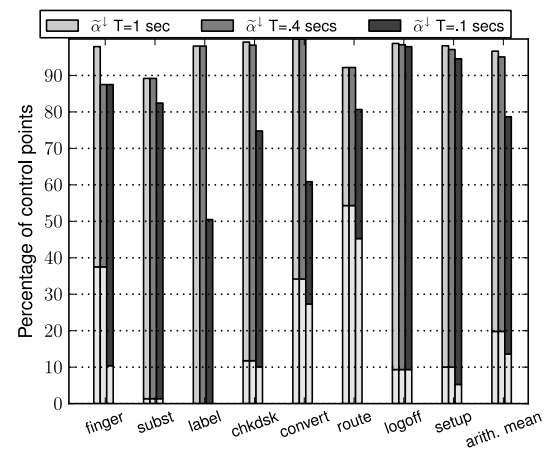

(b)

Fig. 6. (a) Performance: $\widetilde{\alpha}^{\downarrow}$ vs. $\widehat{\alpha}^{\uparrow}$. (b) Precision: \% of control points at which $\widetilde{\alpha}^{\downarrow}$ has as good or better precision as $\widehat{\alpha}^{\uparrow}$; the lighter-color lower portion of each bar indicates the $\%$ of control points at which the precision is strictly greater for $\widetilde{\alpha}^{\downarrow}$.

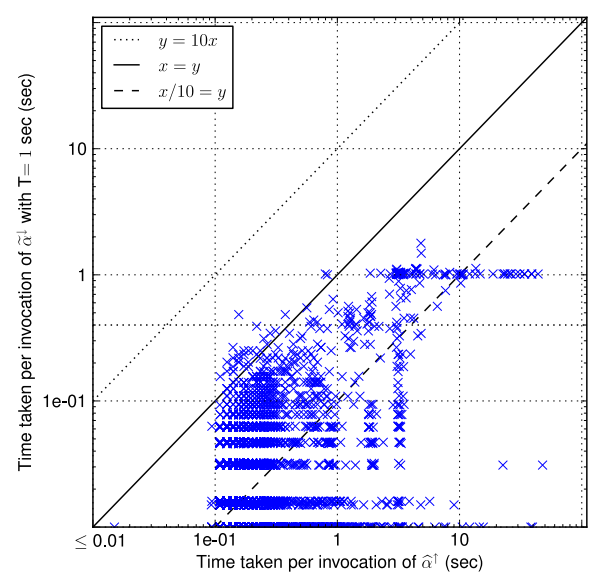

(a)

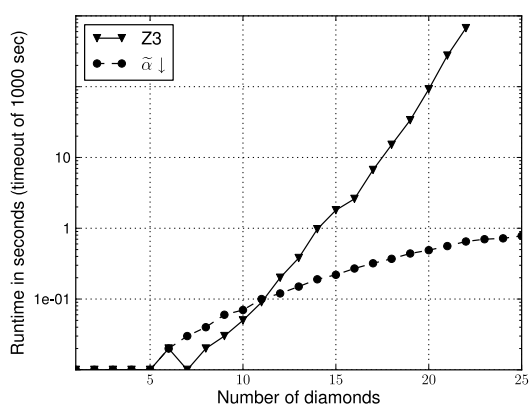

(b)

Fig. 7. (a) Log-log scatter plot of transformer-construction time. (b) Semilog plot of Z3 vs. $\widetilde{\alpha}^{\downarrow}$ on $\chi_{d}$ formulas.

of the SMT solver times out, $\widehat{\alpha}^{\uparrow}$ is forced to return $\top_{K S}$ in order to be sound. (Consequently, it is possible for $\widetilde{\alpha}^{\downarrow}$ to return a more precise answer than $\widehat{\alpha}^{\uparrow}$.)

The setup for the $\widetilde{\alpha}^{\downarrow}$-based analysis is the same as the baseline $\widehat{\alpha}^{\uparrow}$-based analysis, except that we call $\widetilde{\alpha}^{\downarrow}$ when calculating the KS weight for a basic block. We use 1-assume in this experiment. Each basic-block formula $\varphi_{B}$ is rewritten to a set of integrity constraints, with ITE-terms rewritten as illustrated in Ex. 1. The priority of a Boolean variable is its postorder-traversal number, and is used to select which variable is used in the Dilemma Rule. We bound the total time taken by each call to $\widetilde{\alpha}^{\downarrow}$ to a fixed timeout T. Note that even when the call to $\widetilde{\alpha}^{\downarrow}$ times out, it can still return a sound non- $T_{K S}$ value. We ran $\widetilde{\alpha}^{\downarrow}$ using $\mathrm{T}=1$ sec, $\mathrm{T}=0.4$ secs, and $\mathrm{T}=0.1$ secs. 
Fig. 6(a) shows the normalized time taken for WPDS construction when using $\widetilde{\alpha}^{\downarrow}$ with $\mathrm{T}=1 \mathrm{sec}, \mathrm{T}=0.4$ secs, and $\mathrm{T}=0.1$ secs. The running time is normalized to the corresponding time taken by $\widehat{\alpha}^{\uparrow}$; lower numbers are better. WPDS construction using $\widetilde{\alpha}^{\downarrow}$ with $\mathrm{T}=1 \mathrm{sec}$. is about 11.3 times faster than $\widehat{\alpha}^{\uparrow}$ (computed as the geometric mean), which answers question 1.

Decreasing the timeout T makes the $\widetilde{\alpha}^{\downarrow}$ WPDS construction only slightly faster: on average, going from $\mathrm{T}=1 \mathrm{sec}$. to $\mathrm{T}=.4$ secs. reduces WPDS construction time by only $17 \%$ (computed as the geometric mean). To understand this behavior better, we show in Fig. 77(a) a log-log scatter-plot of the times taken by $\widehat{\alpha}^{\uparrow}$ versus the times taken by $\widetilde{\alpha}^{\downarrow}$ (with $\mathrm{T}=1 \mathrm{sec}$.), to generate the transformers for each basic block in the benchmark suite. As shown in Fig. 7(a), the times taken by $\widetilde{\alpha}^{\downarrow}$ are bounded by 1 second. (There are a few calls that take more than 1 second; they are an artifact of the granularity of operations at which we check whether the procedure has timed out.) Most of the basic blocks take less than 0.4 seconds, which explains why the overall time for WPDS construction does not decrease much as we decrease T in Fig. 6(a). We also see that the $\widehat{\alpha}^{\uparrow}$ times are not bounded, and can be as high as 50 seconds.

To answer question 2 we compared the precision of the WPDS analysis when using $\widetilde{\alpha}^{\downarrow}$ with T equal to $1,0.4$, and 0.1 seconds with the precision obtained using $\widehat{\alpha}^{\uparrow}$. In particular, we compare the affine relations (i.e., invariants) computed by the $\widetilde{\alpha}^{\downarrow}$-based and $\widehat{\alpha}^{\uparrow}$-based analyses for each control point-i.e., the beginning of a basic block that ends with a branch. Fig. 6(b) shows the percentage of control points for which the $\widetilde{\alpha}^{\downarrow}$-based analysis computes a better (tighter) or equally precise affine relation. On average, when using $\mathrm{T}=1 \mathrm{sec}, \widetilde{\alpha}^{\downarrow}$-based analysis computes an equally precise invariant at $76.9 \%$ of the control points (computed as the arithmetic mean). Interestingly, the $\widetilde{\alpha}^{\downarrow}$-based analysis computes an answer that is more precise compared to that computed by the $\widehat{\alpha}^{\uparrow}$-based analysis. That is not a bug in our implementation; it happens because $\widehat{\alpha}^{\uparrow}$ has to return $\top_{\mathrm{KS}}$ when the call to the SMT solver times out. In Fig. 6(b), the lighter-color lower portion of each bar shows the percentage of control points for which $\widetilde{\alpha}^{\downarrow}$-based analysis provides strictly more precise invariants when compared to $\widehat{\alpha}^{\uparrow}$-based analysis; on average, $\widetilde{\alpha}^{\downarrow}$-based analysis is more precise for $19.8 \%$ of the control points (arithmetic mean, for $\mathrm{T}=1$ second). $\widetilde{\alpha}^{\downarrow}$-based analysis is less precise at only $3.3 \%$ of the control points. Furthermore, as expected, when the timeout for $\widetilde{\alpha}^{\downarrow}$ is reduced, the precision decreases.

Satisfiability Checking. The formula used in Ex. 2 is just one instance of a family of unsatisfiable QF_LRA formulas [25]. Let $\chi_{d}=\left(a_{d}<a_{0}\right) \wedge \bigwedge_{i=0}^{d-1}\left(\left(a_{i}<\right.\right.$ $\left.\left.b_{i}\right) \wedge\left(a_{i}<c_{i}\right) \wedge\left(\left(b_{i}<a_{i+1}\right) \vee\left(c_{i}<a_{i+1}\right)\right)\right)$. The formula $\psi$ in Ex. 2 is $\chi_{2}$; that is, the number of "diamonds" is 2 (see Fig. 1 (a)). We used the QF_LRA/Polyhedra instantiation of our framework to check whether $\widetilde{\alpha}\left(\chi_{d}\right)=\perp$ for $d=1 \ldots 25$ using 1-assume. We ran this experiment on a single processor of a 16-core 2.4 $\mathrm{GHz}$ Intel Zeon computer running 64-bit RHEL Server release 5.7. The semilog plot in Fig. 7(b) compares the running time of $\widetilde{\alpha}^{\downarrow}$ with that of Z3, version 3.2 [1]. The time taken by Z3 increases exponentially with $d$, exceeding the timeout threshold of 1000 seconds for $d=23$. This corroborates the results of a 
similar experiment conducted by McMillan et al. 25], where the reader can also find an in-depth explanation of this behavior.

On the other hand, the running time of $\widetilde{\alpha}^{\downarrow}$ increases linearly with $d$ taking 0.78 seconds for $d=25$. The cross-over point is $d=12$. In Ex. 2, we saw how two successive applications of the Dilemma Rule suffice to prove that $\psi$ is unsatisfiable. That explanation generalizes to $\chi_{d}: d$ applications of the Dilemma Rule are sufficient to prove unsatisfiability of $\chi_{d}$. The order in which Boolean variables with unknown truth values are selected for use in the Dilemma Rule has no bearing on this linear behavior, as long as no variable is starved from being chosen (i.e., a fair-choice schedule is used). Each application of the Dilemma Rule is able to infer that $a_{i}<a_{i+1}$ for some $i$.

We do not claim that $\widetilde{\alpha}^{\downarrow}$ is better than mature SMT solvers such as Z3. We do believe that it represents another interesting point in the design space of SMT solvers, similar in nature to the GDPLL algorithm [25] and the $k$-lookahead technique used in the DPLL(ப) algorithm [4.

\section{Applications to Other Symbolic Operations}

The symbolic operations of $\widehat{\gamma}$ and $\widehat{\alpha}$ can be used to implement a number of other useful operations, as discussed below. In each case, over-approximations result if $\widehat{\alpha}$ is replaced by $\widetilde{\alpha}$.

- The operation of containment checking, $A_{1} \sqsubseteq A_{2}$, which is needed by analysis algorithms to determine when a post-fixpoint is attained, can be implemented by checking whether $\widehat{\alpha}\left(\widehat{\gamma}\left(A_{1}\right) \wedge \neg \widehat{\gamma}\left(A_{2}\right)\right)$ equals $\perp$.

- Suppose that there are two Galois connections $\mathcal{G}_{1}=\mathcal{C} \underset{\alpha_{1}}{\stackrel{\gamma_{1}}{\leftrightarrows}} \mathcal{A}_{1}$ and $\mathcal{G}_{2}=\mathcal{C} \underset{\alpha_{2}}{\stackrel{\gamma_{2}}{\leftrightarrows}} \mathcal{A}_{2}$, and one wants to work with the reduced product of $\mathcal{A}_{1}$ and $\mathcal{A}_{2}$ [7, $\left.\S 10.1\right]$. The semantic reduction of a pair $\left(A_{1}, A_{2}\right)$ can be performed by letting $\psi$ be the formula $\widehat{\gamma}_{1}\left(A_{1}\right) \wedge \widehat{\gamma}_{2}\left(A_{2}\right)$, and creating the pair $\left(\widehat{\alpha}_{1}(\psi), \widehat{\alpha}_{2}(\psi)\right)$.

- Given $A_{1} \in \mathcal{A}_{1}$, one can find the most-precise value $A_{2} \in \mathcal{A}_{2}$ that overapproximates $A_{1}$ in $\mathcal{A}_{2}$ as follows: $A_{2}=\widehat{\alpha}_{2}\left(\widehat{\gamma}_{1}\left(A_{1}\right)\right)$.

- Given a loop-free code fragment $F$, consisting of one or more blocks of program statements and conditions, one can obtain a representation of its best transformer by symbolically executing $F$ to obtain a transition formula $\psi_{F}$, and then performing $\widehat{\alpha}\left(\psi_{F}\right)$.

\section{Related Work}

Extensions of Stålmarck's Method. Björk [3] describes extensions of Stålmarck's method to first-order logic. Like Björk, our work goes beyond the classical setting of Stålmarck's method [33] (i.e., propositional logic) and extends the method to more expressive logics, such as QF_LRA or QF_BV. However, Björk is concerned solely with validity checking, and-compared with the 
propositional case - the role of abstraction is less clear in his method. Our algorithm not only uses an abstract domain as an explicit datatype, the goal of the algorithm is to compute an abstract value $A^{\prime}=\widehat{\operatorname{Assume}}[\varphi](A)$.

Our approach was influenced by Granger's method of using (in)equation solving as a way to implement semantic reduction and Assume as part of his technique of local decreasing iterations [16]. Granger describes techniques for performing reductions with respect to (in)equations of the form $x_{1} \bowtie F\left(x_{1}, \ldots, x_{n}\right)$ and $\left(x_{1} * F\left(x_{1}, \ldots, x_{n}\right)\right) \bowtie G\left(x_{1}, \ldots, x_{n}\right)$, where $\bowtie$ stands for a single relational symbol of $\mathcal{L}$, such as $=, \neq,<, \leq,>, \geq$, or $\equiv$ (arithmetical congruence). Our framework is not limited to literals of these forms; all that we require is that for a literal $l \in \mathcal{L}$, there is an algorithm to compute an overapproximating value $\mu \widetilde{\alpha}(l)$. Moreover, Granger has no analog of the Dilemma Rule, nor does he present any completeness results (cf. \$4).

SMT Solvers. Most methods for SMT solving can be classified according to whether they employ lazy or eager translations to SAT. (The SAT procedure then employed is generally based on the DPLL procedure 109.) In contrast, the algorithm for SMT described in this paper is not based on a translation to SAT; instead, it generalizes Stålmarck's method for propositional logic to richer logics.

Lazy approaches abstract each atom of the input formula to a distinct propositional variable, use a SAT solver to find a propositional model, and then check that model against the theory [11311]. The disadvantage of the lazy approach is that it cannot use theory information to prune the search. In contrast, our algorithm is able to use theory-specific information to make deductions - in particular, in the LeafRule function (Fig. 4) used in Alg. 2. The use of theory-specific information is the reason why our approach outperformed Z3, which uses the lazy approach, on the diamond example (\$5).

Eager approaches 534 encode more of the theory into the propositional formula that is given to the SAT solver, and hence are able to constrain the solution space with theory-specific information. The challenge in designing such solvers is to ensure that the propositional formula does not blow up in size. In our approach, such an explosion in the set of literals in the formula is avoided because our learned facts are restricted by the abstract domain in use.

A variant of the Dilemma Rule is used in DPLL( $\sqcup)$, and allows the theory solver in a lazy DPLL-based SMT solver to produce joins of facts deduced along different search paths. However, as pointed out by Bjørner et al. [4, §5], their system is weaker than Stålmarck's method, because Stålmarck's method can learn equivalences between literals.

Another difference between our work and existing approaches to SMT is the connection presented in this paper between Stålmarck's method and the computation of best abstract operations for abstract interpretation.

Best Abstract Operations. Several papers about best abstract operations have appeared in the literature [14,29,3721,12]. Graf and Saïdi [14] showed that decision procedures can be used to generate best abstract transformers for predicate-abstraction domains. Other work has investigated more efficient 
methods to generate approximate transformers that are not best transformers, but approach the precision of best transformers [26].

Several techniques work from below 292112 -performing joins to incorporate more and more of the concrete state space - which has the drawback that if they are stopped before the final answer is reached, the most-recent approximation is an under-approximation of the desired value. In contrast, our technique works from above. It can stop at any time and return a safe answer.

Yorsh et al. [37 developed a method that works from above to perform Assume $[\varphi](A)$ for the kind of abstract domains used in shape analysis (i.e., "canonical abstraction" of logical structures [30]). Their method has a splitting step, but no analog of the join step performed at the end of an invocation of the Dilemma Rule. In addition, their propagation rules are much more heavyweight.

Template Constraint Matrices (TCMs) are a parametrized family of linearinequality domains for expressing invariants in linear real arithmetic. Sankaranarayanan et al. 31] gave a parametrized meet, join, and set of abstract transformers for all TCM domains. Monniaux [26] gave an algorithm that finds the best transformer in a TCM domain across a straight-line block (assuming that concrete operations consist of piecewise linear functions), and good transformers across more complicated control flow. However, the algorithm uses quantifier elimination, and no polynomial-time elimination algorithm is known for piecewise-linear systems.

Cover Algorithms. Gulwani and Musuvathi [17] defined the "cover problem", which addresses approximate existential quantifier elimination: Given a formula $\varphi$ in $\operatorname{logic} \mathcal{L}$, and a set of variables $V$, find the strongest quantifier-free formula $\bar{\varphi}$ in $\mathcal{L}$ such that $\llbracket \exists V: \varphi \rrbracket \subseteq \llbracket \bar{\varphi} \rrbracket$. They presented cover algorithms for the theories of uninterpreted functions and linear arithmetic, and showed that covers exist in some theories that do not support quantifier elimination.

The notion of a cover has similarities to the notion of symbolic abstraction, but the two notions are distinct. Our technical report 36] discusses the differences in detail, describing symbolic abstraction as over-approximating a formula $\varphi$ using an impoverished logic fragment, while a cover algorithm only removes variables $V$ from the vocabulary of $\varphi$. The two approaches yield different overapproximations of $\varphi$, and the over-approximation obtained by a cover algorithm does not, in general, yield suitable abstract values and abstract transformers.

\section{References}

1. Armando, A., Castellini, C., Giunchiglia, E.: SAT-Based Procedures for Temporal Reasoning. In: Biundo, S., Fox, M. (eds.) ECP 1999. LNCS, vol. 1809, pp. 97-108. Springer, Heidelberg (2000)

2. Ball, T., Podelski, A., Rajamani, S.K.: Boolean and Cartesian Abstraction for Model Checking C Programs. In: Margaria, T., Yi, W. (eds.) TACAS 2001. LNCS, vol. 2031, pp. 268-283. Springer, Heidelberg (2001)

3. Björk, M.: First order Stålmarck. J. Autom. Reasoning 42(1), 99-122 (2009)

4. Bjørner, N., de Moura, L.: Accelerated lemma learning using joins-DPLL( $\sqcup)$. In: LPAR (2008) 
5. Bryant, R.E., Velev, M.N.: Boolean satisfiability with transitivity constraints. Trans. on Computational Logic 3(4) (2002)

6. Clarke, E., Kroening, D., Sharygina, N., Yorav, K.: Predicate abstraction of ANSIC programs using SAT. FMSD 25(2-3) (2004)

7. Cousot, P., Cousot, R.: Systematic design of program analysis frameworks. In: POPL (1979)

8. Cousot, P., Halbwachs, N.: Automatic discovery of linear constraints among variables of a program. In: POPL (1978)

9. Davis, M., Logemann, G., Loveland, D.: A machine program for theorem-proving. Commun. ACM 5(7) (1962)

10. Davis, M., Putnam, H.: A computing procedure for quantification theory. J. ACM 7(3) (1960)

11. de Moura, L., Bjørner, N.: Z3: An Efficient SMT Solver. In: Ramakrishnan, C.R., Rehof, J. (eds.) TACAS 2008. LNCS, vol. 4963, pp. 337-340. Springer, Heidelberg (2008)

12. Elder, M., Lim, J., Sharma, T., Andersen, T., Reps, T.: Abstract Domains of Affine Relations. In: Yahav, E. (ed.) SAS 2011. LNCS, vol. 6887, pp. 198-215. Springer, Heidelberg (2011)

13. Flanagan, C., Joshi, R., Ou, X., Saxe, J.B.: Theorem Proving Using Lazy Proof Explication. In: Hunt Jr., W.A., Somenzi, F. (eds.) CAV 2003. LNCS, vol. 2725, pp. 355-367. Springer, Heidelberg (2003)

14. Graf, S., Saïdi, H.: Construction of Abstract State Graphs with PVS. In: Grumberg, O. (ed.) CAV 1997. LNCS, vol. 1254, pp. 72-83. Springer, Heidelberg (1997)

15. Graham, S., Wegman, M.: A fast and usually linear algorithm for data flow analysis. J. ACM 23(1), 172-202 (1976)

16. Granger, P.: Improving the Results of Static Analyses Programs by Local Decreasing Iteration. In: Shyamasundar, R.K. (ed.) FSTTCS 1992. LNCS, vol. 652, pp. 68-79. Springer, Heidelberg (1992)

17. Gulwani, S., Musuvathi, M.: Cover Algorithms and Their Combination. In: Gairing, M. (ed.) ESOP 2008. LNCS, vol. 4960, pp. 193-207. Springer, Heidelberg (2008)

18. Harrison, J.: Stålmarck's Algorithm as a HOL Derived Rule. In: von Wright, J., Harrison, J., Grundy, J. (eds.) TPHOLs 1996. LNCS, vol. 1125, pp. 221-234. Springer, Heidelberg (1996)

19. Karr, M.: Affine relationship among variables of a program. Acta Inf. 6 (1976)

20. Kidd, N., Lal, A., Reps, T.: WALi: The Weighted Automaton Library (2007), www.cs.wisc.edu/wpis/wpds/download.php

21. King, A., Søndergaard, H.: Automatic Abstraction for Congruences. In: Barthe, G., Hermenegildo, M. (eds.) VMCAI 2010. LNCS, vol. 5944, pp. 197-213. Springer, Heidelberg (2010)

22. Knoop, J., Steffen, B.: Interprocedural Coincidence Theorem. In: Pfahler, P., Kastens, U. (eds.) CC 1992. LNCS, vol. 641, pp. 125-140. Springer, Heidelberg (1992)

23. Lal, A., Reps, T.: Improving Pushdown System Model Checking. In: Ball, T., Jones, R.B. (eds.) CAV 2006. LNCS, vol. 4144, pp. 343-357. Springer, Heidelberg (2006)

24. Lal, A., Reps, T., Balakrishnan, G.: Extended Weighted Pushdown Systems. In: Etessami, K., Rajamani, S.K. (eds.) CAV 2005. LNCS, vol. 3576, pp. 434-448. Springer, Heidelberg (2005)

25. McMillan, K.L., Kuehlmann, A., Sagiv, M.: Generalizing DPLL to Richer Logics. In: Bouajjani, A., Maler, O. (eds.) CAV 2009. LNCS, vol. 5643, pp. 462-476. Springer, Heidelberg (2009)

26. Monniaux, D.: Automatic modular abstractions for template numerical constraints. LMCS 6(3) (2010) 
27. Müller-Olm, M., Seidl, H.: Analysis of modular arithmetic. TOPLAS (2007)

28. PPL: The Parma polyhedra library, http://www.cs.unipr.it/ppl/

29. Reps, T., Sagiv, M., Yorsh, G.: Symbolic Implementation of the Best Transformer. In: Steffen, B., Levi, G. (eds.) VMCAI 2004. LNCS, vol. 2937, pp. 252-266. Springer, Heidelberg (2004)

30. Sagiv, M., Reps, T., Wilhelm, R.: Parametric shape analysis via 3 -valued logic. TOPLAS 24(3), 217-298 (2002)

31. Sankaranarayanan, S., Sipma, H.B., Manna, Z.: Scalable Analysis of Linear Systems Using Mathematical Programming. In: Cousot, R. (ed.) VMCAI 2005. LNCS, vol. 3385 , pp. 25-41. Springer, Heidelberg (2005)

32. Sharir, M., Pnueli, A.: Two approaches to interprocedural data flow analysis. In: Program Flow Analysis: Theory and Applications. Prentice-Hall (1981)

33. Sheeran, M., Stålmarck, G.: A tutorial on Stålmarck's proof procedure for propositional logic. FMSD 16(1) (2000)

34. Strichman, O.: On Solving Presburger and Linear Arithmetic with SAT. In: Aagaard, M.D., O'Leary, J.W. (eds.) FMCAD 2002. LNCS, vol. 2517, pp. 160-170. Springer, Heidelberg (2002)

35. Thakur, A., Reps, T.: A generalization of Stålmarck's method. TR 1699. CS Dept., Univ. of Wisconsin, Madison, WI (October 2011)

36. Thakur, A., Reps, T.: A method for symbolic computation of precise abstract operations. TR 1708. CS Dept., Univ. of Wisconsin, Madison, WI (January 2012)

37. Yorsh, G., Reps, T., Sagiv, M.: Symbolically Computing Most-Precise Abstract Operations for Shape Analysis. In: Jensen, K., Podelski, A. (eds.) TACAS 2004. LNCS, vol. 2988, pp. 530-545. Springer, Heidelberg (2004) 